\title{
Yes, Gauss's Answer IS InDEED CORRECT!
}

\author{
Zafer Ercan, Mehmet Vural \\ Department of Mathematics, Abant İzzet Baysal University, Turkey \\ zercan@ibu.edu.tr, m.vural.hty@gmail.com
}

Abstract: A meaning of three dots (...) and the Gauss's sum.

Keywords: three dots, Gauss sum.

\section{Gauss's Answer}

One day when Carl Friedrich Gauss was a primary school student, the teacher asked the students to find the sum of the numbers from one to one hundred to silence the noisy class. A couple of minutes later, the teacher noticed that Gauss started to get interested with something different. As soon as he warned the young pupil to keep on working on the problem he had assigned, Gauss told the teacher that he had already had the answer [1. Gauss's ultimately elegant method to find the sum is as follows:

Letting

$$
x=1+2+\ldots+100
$$

and observing that

$$
x=100+99+\ldots+1,
$$

one has

$$
\begin{aligned}
2 x & =x+x \\
& =(1+2+\ldots+100)+(100+99+\ldots+1) \\
& =(1+100)+(2+99)+\ldots+(100+1) \\
& =101+101+\ldots+101 \\
& =101 \ldots 100 \\
& =10100,
\end{aligned}
$$

which, upon dividing by two, yields $x=5050$. Although the answer is definitely correct, there is a "gap" in the proof, since the three dots (...) appearing in the above solution are not mathematically defined although their 
meaning is clear and using them makes perfectly sense intuitively. Yet, had the teacher asked to "find the sum of one hundred times zero," any solution to it provided in the way of the above one would have included a gap as such!

The aim of this short note is to attach a "meaning", more precisely to give a mathematical definition, to three dots (...).

\section{Odds and ends}

A set is called transitive if every element of it is also a subset of it. A transitive set is called an ordinal if it is totally ordered by set inclusion. Following the construction of the set of natural numbers in the Zermelo-Fraenkel set theory, we consider each natural number as an ordinal. The set of natural numbers is denoted by $\omega$. The function $s: \omega \rightarrow \omega$ defined by $s(n)=n \cup\{n\}$ is called the successor of $n$. Note that $s(n)=n+1$. In particular,

$$
s(n)=\{k \in \omega: 0 \leqslant k \leqslant n\}=\{k \in \omega: 0 \leqslant k<n+1\}
$$

hold. If there is a bijection between two natural numbers, then they are equal. Two sets are called equivalent if there exists a bijection between them. A set $A$ is called finite if there exists a bijection $f: n \rightarrow A$ for some $n \in \omega$, in which case one writes $n=|A|$. In particular, for each $n \in \omega$, one has $n=|n|$. The union of the family $\left(A_{i}\right)_{i \in I}$ of disjoint sets is denoted by $\dot{\bigcup}_{i \in I} A_{i}$. As an instance, if $\left\{A_{k}: 0 \leqslant k \leqslant n\right\}$ is a set whose elements are pairwisely disjoint, the union of this set is denoted by $\dot{U}_{0 \leqslant k \leqslant n} A_{k}$.

\section{Meaning of three dots: From intuitive to mathematical}

Definition. Let $n \in \omega$ and $f: n \rightarrow \omega$ be a function. The cardinal number of the union of the set $\{f(k) \times\{k\}: k \in n\}$ is said to be the sum of the function $f$ and is denoted by $\oplus f$.

To examplify, let $f: 2 \rightarrow \omega$ be defined by $f(0)=2, f(1)=1$. Then

$$
\begin{aligned}
\oplus f & =\left|\dot{\cup}_{k \in 2} f(k) \times\{k\}\right| \\
& =|(f(0) \times\{0\}) \dot{\cup}(f(1) \times\{1\})| \\
& =|f(0) \times\{0\}|+|f(1) \times\{1\}| \\
& =2+1 \\
& =3 .
\end{aligned}
$$


Theorem 1. If $n \in \omega, \mathbf{1}_{n}: n \rightarrow \omega$ is the constant one function, i.e., $f(k)=1$ for all $k \in n$, then $\oplus f=n$.

Proof. We will use induction.

Let

$$
A=\left\{n \in \omega: \oplus \mathbf{1}_{n}=n\right\}
$$

Since

$$
\mathbf{1}_{0}=\left|\oplus \bigcup_{k \in 0} f(k) \times\{k\}\right|=|\emptyset|=0,
$$

we get $0 \in A$. Suppose now that $n \in A$. Therefore we have

$$
\begin{aligned}
\oplus \mathbf{1}_{s(n)} & =\left|\dot{\bigcup}_{k \in s(n)} \mathbf{1}_{s(n)}(k) \times\{k\}\right| \\
& =\left|\left(\bigcup_{k \in n} \mathbf{1}_{s(n)}(k) \times\{k\}\right) \dot{\bigcup} 1 \times\{n\}\right| \\
& =\left|\left(\bigcup_{k \in n} \mathbf{1}_{n} \times\{k\}\right)\right|+|1 \times\{n\}| \\
& =\oplus \mathbf{1}_{n}+1 \\
& =n+1 \\
& =s(n)
\end{aligned}
$$

It then follows that $s(n) \in A$, whence, by induction, one gets $A=\omega$. This completes the proof.

Let $n \in \omega$ and let $f$ and $g$ be functions from $n$ into $\omega$. The function $f+g$ (sum of $f$ and $g$ ) from $n$ into $w$ is defined by

$$
(f+g)(n)=f(n)+g(n) .
$$

Theorem 2. Let $f, g: n \rightarrow \omega$ be two functions. Then

$$
\oplus(f+g)=\oplus f+\oplus g .
$$

Proof. Observe that for each $k \in \dot{U} \omega$, we have

$$
\begin{aligned}
\dot{\bigcup}_{k \in n}[f(k)+g(k)] \times\{k\} & =\dot{\bigcup}_{k \in n}[(f(k) \times\{0\}) \dot{U}(g(k) \times\{1\})] \times\{k\} \\
& =\left[\bigcup_{k \in n}[((f(k) \times\{0\}) \times\{k\}) \dot{U}((g(k) \times\{1\}) \times\{k\})]\right. \\
& =\left[\bigcup _ { k \in n } [ ( ( f ( k ) \times \{ 0 \} ) \times \{ k \} ) ] \dot { \cup } \left[\dot{\bigcup}_{k \in n}[((g(k) \times\{1\}) \times\{k\})]\right.\right.
\end{aligned}
$$

This implies

$$
\begin{aligned}
\oplus(f+g) & =\mid \bigcup_{k \in n}[(f(k)+g(k)] \times\{k\} \mid \\
& =\mid\left[\bigcup _ { k \in n } [ ( ( f ( k ) \times \{ 0 \} ) \times \{ k \} ) ] | + | \left[\dot{\bigcup}_{k \in n}[((g(k) \times\{1\}) \times\{k\})] \mid\right.\right. \\
& =\mid\left[\dot { \bigcup } _ { k \in n } \left[( ( f ( k ) \times \{ k \} ) ] | + | \left[\dot{\bigcup}_{k \in n}[((g(k) \times\{k\})] \mid\right.\right.\right. \\
& =(\oplus f)+(\oplus g),
\end{aligned}
$$

whence the result.

For $m \in \omega$ and a function $f: n \rightarrow \omega$, the function $m f$ from $n$ into $\omega$ is defined by

$$
(m f)(n)=m f(n)
$$


Theorem 3. Let $f: n \rightarrow \omega$ be a function and $m \in \omega$. Define $m f: n \rightarrow \omega$ by $(m f)(k)=m f(k)$. Then

$$
\oplus m f=m(\oplus f)
$$

Proof. Follows from performing induction over $n$.

Let $n \in \omega$. If $f: n \rightarrow \omega$ a function and $g: n \rightarrow n$ is a bijection function then the composition function $f \circ g$ of $f$ and $g$ from $n$ into $\omega$ defined by

$$
f \circ g(n)=f(g(n))
$$

The proof of the following is straightforward.

Theorem 4. Let $f: n \rightarrow \omega$ be a function and $i: n \rightarrow n$ be a bijection. Then

$$
\oplus f=\oplus(f \circ i)
$$

It turns out, in the light of what has obtained above, that the question asked to young Gauss by his teacher is the following one: Let $f: s(100) \rightarrow \omega$ be defined by $f(k)=k$. Find the sum $\oplus f$. And the answer to this question is now apparent: Let $i: s(100) \rightarrow n$ be defined by $i(k)=100-k$ (that is, $i(k)+k=100)$. Let $g=f \circ i$. Then $f+g: s(100) \rightarrow \omega$, the sum of $f$ and $g$, is defined by $(f+g)(k)=100$, so that, by using the above theorems, one can have

$$
\begin{aligned}
2 \oplus f & =(\oplus f)+(\oplus f) \\
& =\oplus f+\oplus g \\
& =\oplus(f+g)=\oplus 100 \mathbf{1}_{s(100)} \\
& =100 \oplus \mathbf{1}_{101} \\
& =100 \cdot 101 .
\end{aligned}
$$

This implies

$$
\oplus f=\frac{100.101}{2}=5050 \text {. }
$$

\section{References}

[1] Waltershausen, W. Gauss zum Gedächtniss (in German), p. 12, 1856. 